\title{
EXISTENCE OF LIPSCHITZIAN SOLUTIONS TO THE CLASSICAL PROBLEM OF THE CALCULUS OF VARIATIONS IN THE AUTONOMOUS CASE
}

\author{
A. CELLINA *, A. FERRIERO \\ Dip. di Matematica e Applicazioni, Università degli Studi di Milano-Bicocca, \\ Via Bicocca degli Arcimboldi 8, 20126 Milan, Italy \\ Received 7 May 2002, accepted 27 September 2002
}

ABSTRACT. - Under general growth assumptions, that include some cases of linear growth, we prove existence of Lipschitzian solutions to the problem of minimizing $\int_{a}^{b} L\left(x(s), x^{\prime}(s)\right) \mathrm{d} s$ with the boundary conditions $x(a)=A, x(b)=B$.

(c) 2003 L'Association Publications de l'Institut Henri Poincaré. Published by Elsevier B.V. All rights reserved

MSC: 49N60; 49J65

Keywords: Calculus of variations; Existence and Lipschitzianity of solutions

RÉSUMÉ. - Dans l'article on démontre l'existence de solutions Lipschitziennes du problème de minimiser $\int_{a}^{b} L\left(x(s), x^{\prime}(s)\right) \mathrm{d} s, x(a)=A, x(b)=B$, avec des conditions faibles, de croissance, qui comprennent des cas de croissance linéaire.

(C) 2003 L'Association Publications de l'Institut Henri Poincaré. Published by Elsevier B.V. All rights reserved

\section{Introduction}

The direct method of the calculus of variations is based on the notions of coercitivity and of weak lower semicontinuity. From the coercitivity of the functional one derives the property that every sequence that makes the values of the functional bounded, in particular, every minimizing sequence, contains a weakly converging subsequence, and the weak lower semicontinuity implies that the minimum is attained on the weak limit of the minimizing subsequence. For the classical problem of the calculus of variations, the minimum is seeked among the absolutely continuous functions assuming given values at the boundary points and the natural norm of this space is the $\mathbf{L}^{1}$ norm of the derivatives. For the space $\mathbf{L}^{1}$, a necessary and sufficient condition for weak pre-compactness of a sequence is expressed by the criterion of De la Vallée Poussin [4], whose application implies that the Lagrangean $L(x, \xi)$, appearing under the integral sign, has to grow

\footnotetext{
* Corresponding author.

E-mail addresses: cellina@matapp.unimib.it (A. Cellina), ferriero@matapp.unimib.it (A. Ferriero).
} 
faster than linearly with respect to the variable $\xi$. A necessary and sufficient condition seems to leave little hope of being able to apply the method to provide an existence theorem for absolutely continuous minimizers under assumptions that do not imply superlinear growth. The purpose of this paper, however, is to show that in the case of autonomous problems, where the Lagrangean does not depend explicitely on the integration variable $t$, a minor variant of the direct method can be applied under more general growth assumptions. More precisely, we consider problem $(\mathbf{P})$, the problem of minimizing the integral

$$
\int_{a}^{b} L\left(x(s), x^{\prime}(s)\right) \mathrm{d} s
$$

for $x:[a, b] \rightarrow \mathbb{R}^{N}$ absolutely continuous and satisfying $x(a)=A, x(b)=B$. Under more general growth conditions, that include the classical superlinear growth but also some cases of Lagrangeans with linear growth, we show that, from any sequence $\left\{x_{n}\right\}_{n \in \mathbb{N}}$, minimizing for the functional, one can derive another sequence $\left\{\bar{x}_{n}\right\}_{n \in \mathbb{N}}$, each function $\bar{x}_{n}$ obtained from $x_{n}$ by reparametrizing the interval $[a, b]$, that is again minimizing, and consists of equi-Lipschitzian functions. As a consequence, in the case the Lagrangean $L(x, \xi)$ is convex in $\xi$, one can prove the existence of a solution to problem $(\mathbf{P})$, that, in particular, is a Lipschitzian function. A result on the regularity (Lipschitzianity) of solutions to autonomous minimum problems, under conditions of superlinear growth, was established in [5] and, under weaker growth conditions, in [2].

The growth assumption we consider is expressed in terms of the polar of the Lagrangean $L$ with respect to $\xi$ (for the properties fo the polar see, e.g., [6]). The same condition was already introduced in [3] to prove existence of solutions for a rather special class of Lagrangeans. The results we present apply to different classes of Lagrangeans, that can possibly be extended valued and either convex or differentiable in $\xi$. A simple example of a convex everywhere defined Lagrangean satisfying the assumptions of our Theorem 1, in particular the growth condition, is the map, having linear growth,

$$
L(\xi)= \begin{cases}|\xi|-\ln (|\xi|), & |\xi| \geqslant 1 \\ 1, & \text { otherwise }\end{cases}
$$

\section{Main results}

In what follows $L(x, \xi): \mathbb{R}^{N} \times \mathbb{R}^{N} \rightarrow \overline{\mathbb{R}}=\mathbb{R} \cup\{+\infty\}$ is an extended valued function, continuous and bounded below, not identically $+\infty . L^{*}(x, p)$ is the polar of $L$ with respect to its second variable [1], i.e.

$$
L^{*}(x, p)=\sup _{\xi \in \mathbb{R}^{N}}\langle p, \xi\rangle-L(x, \xi)
$$

We denote by dom $=\left\{(x, \xi) \in \mathbb{R}^{N} \times \mathbb{R}^{N}: L(x, \xi) \in \mathbb{R}\right\}$ its effective domain. Since the assumptions on $L$ for the case where dom $=\mathbb{R}^{N} \times \mathbb{R}^{N}$ are somewhat simpler than the assumptions needed in the general case, we shall state separately the results for the two cases. For each case, $L$, as a function of $\xi$, may be either convex or not; in this second 
case, we shall need the extra assumption of differentiability of $L$ with respect to $\xi$. This assumption is not needed in the convex case, since, in this case, the existence of a subdifferential is enough for the proof. Hence, we will provide four different statements of what is basically the same result; the proof will be one proof for the four different theorems. We first present the results for the simpler case where dom $=\mathbb{R}^{N} \times \mathbb{R}^{N}$.

THEOREM 1 (Convex case). - Assume that:

(1) dom $=\mathbb{R}^{N} \times \mathbb{R}^{N}$ and $L(x, \cdot)$ is convex, $\forall x \in \mathbb{R}^{N}$;

(2) for every selection $p(x, \cdot) \in \partial_{\xi} L(x, \cdot)$ we have

$$
L^{*}(x, p(x, \xi)) \rightarrow+\infty
$$

as $|\xi|$ tends to $+\infty$, uniformly in $x$.

Then: given any minimizing sequence $\left\{x_{n}\right\}_{n \in \mathbb{N}}$ for the functional in $(\mathbf{P})$, there exists a constant $\Lambda$ and a sequence of reparametrizations $s_{n}$ of the interval $[a, b]$ onto itself, such that $\left\{\bar{x}_{n}\right\}_{n \geqslant n_{1}}=\left\{x_{n} \circ s_{n}\right\}_{n \geqslant n_{1}}$ is again a minimizing sequence and each $\bar{x}_{n}$ is Lipschitzian with Lipschitz constant $\Lambda$.

The convex Lagrangean $L(\xi)$ described in Section 1 is such that

$$
L^{*}(p(\xi))=\ln (|\xi|)-1 \rightarrow+\infty .
$$

THEOREM 2 (Differentiable case). - Assume that:

(1) dom $=\mathbb{R}^{N} \times \mathbb{R}^{N}$ and $\forall x \in \mathbb{R}^{N}, L(x, \cdot)$ is differentiable;

(2) $L^{*}\left(x, \nabla_{\xi} L(x, \xi)\right) \rightarrow+\infty$ as $|\xi|$ tends to $+\infty$, uniformly in $x$.

Then the conclusion of Theorem 1 holds.

The following are the analogous results in the more complex case where dom $\neq$ $\mathbb{R}^{N} \times \mathbb{R}^{N}$. In this case it is not necessarily true that the functional in $(\mathbf{P})$ is not identically $+\infty$.

THEOREM 3 (Convex case). - Assume that:

(1) $L(x, \cdot)$ is a convex extended valued map and $(x, 0) \in$ dom whenever there exists $\xi$ such that $(x, \xi) \in$ dom;

(2) for every selection $p(x, \cdot) \in \partial_{\xi} L(x, \cdot)$ we have

$$
L^{*}(x, p(x, \xi)) \rightarrow+\infty
$$

as $|\xi|$ tends to $+\infty$, with $(x, \xi) \in$ dom, uniformly in $x$;

(3) for every $M>0, \exists \delta>0$ such that $L(x, \xi)>M$, for every $(x, \xi) \in$ dom with $d((x, \xi), \partial \mathrm{dom})<\delta$

(4) the functional in (P) is not identically $+\infty$.

Then the conclusion of Theorem 1 holds.

THEOREM 4 (Differentiable case). - Assume that:

(1) $L(x, \cdot)$ is differentiable and $\operatorname{dom} \cap\left(\{x\} \times \mathbb{R}^{N}\right)$ is star shaped with respect to $(x, 0)$ whenever there exists $\xi$ such that $(x, \xi) \in$ dom;

(2) $L^{*}\left(x, \nabla_{\xi} L(x, \xi)\right) \rightarrow+\infty$ as $|\xi|$ tends to $+\infty$, with $(x, \xi) \in$ dom, uniformly in $x$; 
(3) for every $M>0, \exists \delta>0$ such that $L(x, \xi)>M$, for every $(x, \xi) \in$ dom with $d((x, \xi), \partial$ dom $)<\delta$

(4) the functional in $(\mathbf{P})$ is not identically $+\infty$.

Then the conclusion of Theorem 1 holds.

We shall need the following proposition on the existence of a lower bound for the Lagrangean $L$ under the conditions stated in any of the theorems above.

PROPOSITION 5. - Let L satisfy assumptions (1) and (2) of any of the Theorems 1, 2, 3 or 4 . Then there exist $\alpha>0$ and $\beta \in \mathbb{R}$ such that $L(x, \xi) \geqslant \alpha|\xi|+\beta, \forall(x, \xi) \in \operatorname{dom}$.

Proof. - Set $\ell=\inf \{L(x, \xi)\}$; assumption (2) implies that there exists $r>0$ such that $-L^{*}(x, p(x, \xi)) \leqslant \ell-1$, for every $(x, \xi) \in$ dom with $|\xi| \geqslant r$, where $p(x, \cdot)$ is either $\nabla_{\xi} L(x, \cdot)$ or any selection from the subdifferential of $L(x, \cdot)$. We claim that we can choose $\alpha=1 /(2 r)$ and $\beta=\ell-1$

Fix $(x, \xi) \in$ dom. When $|\xi| \leqslant r$, we have $L(x, \xi) \geqslant \ell>|\xi| /(2 r)+\ell-1$, and the claim is true in this case.

Consider the case $|\xi|>r$. Set $\psi(s)=s /(2 r)+\ell-1$; assumption (1) implies that the convex function $\mathcal{L}(s)=L(x, s \xi /|\xi|)$ is well defined for $s \in[r,|\xi|]$, hence the selection $p_{\mathcal{L}}(s)=\langle\xi /|\xi|, p(x, s \xi /|\xi|)\rangle \in \partial \mathcal{L}(s)$ is increasing and we have $p_{\mathcal{L}}(s) \geqslant$ $p_{\mathcal{L}}(r)$; moreover, from the inequality

$$
L\left(x, r \frac{\xi}{|\xi|}\right)-\left\langle r \frac{\xi}{|\xi|}, p\left(x, r \frac{\xi}{|\xi|}\right)\right\rangle \leqslant \ell-1,
$$

we obtain $p_{\mathcal{L}}(r) \geqslant 1 / r>1 /(2 r)=\psi^{\prime}(s)$. From $\mathcal{L}(r)>\psi(r)$, we obtain $\mathcal{L}(s)>\psi(s)$, for every $s \in[r,|\xi|]$; setting $s=|\xi|$, the claim is proved.

Now, assume the validity of (1), (2) of the differentiable cases. Again, let $r>0$ be such that for every $(x, \xi) \in$ dom with $|\xi| \geqslant r$ we have $-L^{*}\left(x, \nabla_{\xi} L(x, \xi)\right) \leqslant \ell-1$. As before, it follows that the claim is true for $(x, \xi) \in \operatorname{dom},|\xi| \leqslant r$. Fix $\xi,|\xi|>r$. By assumption (1), $\mathcal{L}(s)$ is defined for $s \in[r,|\xi|]$, and we infer

$$
L\left(x, s \frac{\xi}{|\xi|}\right)-\left\langle s \frac{\xi}{|\xi|}, \nabla_{\xi} L\left(x, s \frac{\xi}{|\xi|}\right)\right\rangle \leqslant \ell-1,
$$

so that

$$
\begin{aligned}
\mathcal{L}(s)-\psi(s) & =L\left(x, s \frac{\xi}{|\xi|}\right)-\left[\frac{s}{2 r}+\ell-1\right] \\
& \leqslant\left\langle s \frac{\xi}{|\xi|}, \nabla_{\xi} L\left(x, s \frac{\xi}{|\xi|}\right)\right\rangle+\frac{s}{2 r}=s\left[\mathcal{L}^{\prime}(s)-\psi^{\prime}(s)\right] .
\end{aligned}
$$

Assume that the set $\{s \in(r,|\xi|]: \mathcal{L}(s)-\psi(s)<0\}$ is non-empty, and let $s_{0}$ be its infimum. By continuity, $\mathcal{L}\left(s_{0}\right)-\psi\left(s_{0}\right)=0$, so that $s_{0}>r$. From the Mean Value Theorem we infer the existence of $s_{1} \in\left(r, s_{0}\right)$ such that $\mathcal{L}^{\prime}\left(s_{1}\right)-\psi^{\prime}\left(s_{1}\right)<0$, that in turn implies $\mathcal{L}\left(s_{1}\right)-\psi\left(s_{1}\right)<0$, a contradiction to the definition of $s_{0}$. Hence $\mathcal{L}(s) \geqslant \psi(s)$, $\forall s \in(r,|\xi|]$, in particular for $s=|\xi|$. 
LEMMA 6. - Let $f: \mathbb{R}^{N} \rightarrow \overline{\mathbb{R}}$ be convex, and such that dom contains the origin. Then, for every $\xi$ in the domain of $f$, the function $f(\xi /(1+\cdot))(1+\cdot)$ from $[0,+\infty)$ to $\mathbb{R}$ is convex. Moreover, there exists a selection $p(\cdot) \in \partial f(\cdot)$ such that

$$
f\left(\frac{\xi}{1+s}\right)(1+s)-f(\xi) \leqslant-s f^{*}\left(p\left(\frac{\xi}{1+s}\right)\right), \quad \forall s \in[0,+\infty) .
$$

Proof. - See the proof in [1].

\section{Proof of Theorems 1-4}

Proof. - Set $m$ be the infimum of the values of

$$
\int_{a}^{b} L\left(x(s), x^{\prime}(s)\right) \mathrm{d} s
$$

for $x$ as in problem (P). Proposition 5 and the assumptions of Theorems 1-4 imply that $m$ is finite. Let $\left\{x_{n}\right\}_{n \in \mathbb{N}}$ be a minimizing sequence for problem $(\mathbf{P})$. By Proposition 5 we obtain that there exists $H>0$ such that $\left\|x_{n}^{\prime}\right\|_{1} \leqslant H$ so that, for every $n$, for every $s$ in $[a, b]$, we have $x_{n}(s) \in B[0, A+H]$.

Next point (a) reaches a conclusion with an argument that differs in the cases where $L$ is or is not extended valued, so the argument is presented separately in the two cases.

(a) $\left(\right.$ Case dom $\left.=\mathbb{R}^{N} \times \mathbb{R}^{N}\right)$ For every $n$, consider the subset of $[a, b]$ defined by

$$
T_{n}^{H}=\left\{s \in[a, b]:\left|x_{n}^{\prime}(s)\right| \leqslant 4 H / 3(b-a)\right\} ;
$$

one verifies that the Lebesgue measure of any such set is larger or equal to $(b-a) / 4$.

Fix $\delta>0$. Since $\left(x_{n}(s),(1+\delta) x_{n}^{\prime}(s)\right) \in B[0, A+H] \times B[0,4(1+\delta) H / 3(b-a)]$, and $L$ is continuous, we infer that: there exists $\mu \in \mathbb{R}$ such that, for every $n \in \mathbb{N}$ and $s \in T_{n}^{H}$,

$$
L\left(x_{n}(s),(1+\delta) x_{n}^{\prime}(s)\right) \frac{1}{1+\delta}-L\left(x_{n}(s), x_{n}^{\prime}(s)\right) \leqslant \mu .
$$

(a) (Case dom $\neq \mathbb{R}^{N} \times \mathbb{R}^{N}$ ) Consider a real positive $M$. Assumption (3) implies that there exists $\delta(M)>0$ such that $L(x, \xi)>M, \forall(x, \xi) \in$ dom with $d((x, \xi), \partial$ dom $)<$ $2 \delta(M)$.

Consider the subsets of $[a, b]$

$$
J_{n}^{\delta(M)}=\left\{s \in[a, b]: d\left(\left(x_{n}(s), x_{n}^{\prime}(s)\right), \partial \operatorname{dom}\right) \geqslant 2 \delta(M)\right\} ;
$$

we have the inequality

$$
\begin{aligned}
\int_{a}^{b} L\left(x_{n}(s), x_{n}^{\prime}(s)\right) \mathrm{d} s & =\int_{J_{n}^{\delta(M)}} L\left(x_{n}(s), x_{n}^{\prime}(s)\right) \mathrm{d} s+\int_{[a, b] \backslash J_{n}^{\delta(M)}} L\left(x_{n}(s), x_{n}^{\prime}(s)\right) \mathrm{d} s \\
& \geqslant\left|J_{n}^{\delta(M)}\right| \ell+\left|[a, b] \backslash J_{n}^{\delta(M)}\right| M=(b-a) M+\left|J_{n}^{\delta(M)}\right|(\ell-M),
\end{aligned}
$$


so that

$$
\liminf _{n \rightarrow+\infty}\left|J_{n}^{\delta(M)}\right| \geqslant \frac{(b-a) M-m}{M-\ell} .
$$

Since $\lim _{M \rightarrow+\infty}[(b-a) M-m] /(M-\ell)=b-a$, we can choose $\bar{M}>0$ such that

$$
[(b-a) \bar{M}-m] /(\bar{M}-\ell)>(b-a) 3 / 4 \text {. }
$$

Set $\delta=\delta(\bar{M})$. We have obtained that there exists $n_{1} \in \mathbb{N}$ such that $n \geqslant n_{1}$ implies

$$
\left|J_{n}^{\delta}\right| \geqslant(b-a) 3 / 4
$$

We have also obtained that the sets $\left\{\left(x_{n}(s),(1+\delta) x_{n}^{\prime}(s)\right): s \in J_{n}^{\delta}\right\}$ are contained in dom. Finally, consider the sets

$$
I_{n}^{H}=\left\{s \in[a, b]:\left|x_{n}^{\prime}(s)\right| \leqslant 4 H /(b-a)\right\} ;
$$

the measure of each $I_{n}^{H}$ is at least $(b-a) 3 / 4$ so that, defining $T_{n}^{H}=I_{n}^{H} \cap J_{n}^{\delta}$, we obtain $\left|T_{n}^{H}\right|=\left|I_{n}^{H} \cap J_{n}^{\delta}\right| \geqslant(b-a) / 4, \forall n \geqslant n_{1}$.

Since $\left(x_{n}(s),(1+\delta) x_{n}^{\prime}(s)\right)$ belongs to $B[0, A+H] \times B[0,4(1+\delta) H /(b-a)] \cap$ $\{(x, \xi) \in \operatorname{dom}: d((x, \xi), \partial \operatorname{dom}) \geqslant \delta\}$, a compact subset of dom and $L$ is continuous on dom, we infer that: there exists $\mu \in \mathbb{R}$ such that, for every $n \in \mathbb{N}$ and $s \in T_{n}^{H}$,

$$
L\left(x_{n}(s),(1+\delta) x_{n}^{\prime}(s)\right) \frac{1}{1+\delta}-L\left(x_{n}(s), x_{n}^{\prime}(s)\right) \leqslant \mu .
$$

(b) Consider a real positive $v$ and set $S_{n}^{v}=\left\{s \in[a, b]:\left|x_{n}^{\prime}(s)\right|>v\right\}$. From $\left\|x_{n}^{\prime}\right\|_{1} \leqslant H$, we easily obtain that both the measure of $S_{n}^{\nu}$ and

$$
\varepsilon_{n}^{v}=\int_{S_{n}^{v}}\left[\frac{\left|x_{n}^{\prime}(s)\right|}{v}-1\right] \mathrm{d} s
$$

converge to 0 as $v \rightarrow+\infty$, uniformly with respect to $n \in \mathbb{N}$.

Consider first the convex case; let $p(x, \cdot) \in \partial_{\xi} L(x, \cdot)$ be the selection provided by Lemma 6. By assumption (2) of this case, ther exists a map $M: \mathbb{N} \rightarrow \mathbb{R}$, $\lim _{v \rightarrow+\infty} M(v)=+\infty$, such that

$$
L^{*}(x, p(x, \xi)) \geqslant M(v)
$$

for every $(x, \xi) \in \operatorname{dom} \cap\left[\mathbb{R}^{N} \times(B[0, v])^{c}\right]$; in particular

$$
L^{*}\left(x_{n}(x), p\left(x_{n}(s), x_{n}^{\prime}(s)\right)\right) \geqslant M(v)
$$

for every $n \in \mathbb{N}$ and $s \in S_{n}^{\nu}$. Analogously, under assumption (2) of the differentiable case, there exists a map $M: \mathbb{N} \rightarrow \mathbb{R}, \lim _{v \rightarrow+\infty} M(v)=+\infty$, such that

$$
L^{*}(x, \nabla L(x, \xi)) \geqslant M(v)
$$


for every $(x, \xi) \in \operatorname{dom} \cap\left[\mathbb{R}^{N} \times(B[0, v])^{c}\right]$; in particular

$$
L^{*}\left(x_{n}(x), \nabla L\left(x_{n}(s), x_{n}^{\prime}(s)\right)\right) \geqslant M(v)
$$

for every $n \in \mathbb{N}$ and $s \in S_{n}^{\nu}$.

Hence, both in the convex and in the differentiable case, we have obtained that there exists an integer $\bar{v}$ such that at once we have $\bar{v} \geqslant 4 H /(b-a), M(\bar{v}) \geqslant(1+\delta) \mu$ and $\varepsilon_{n}^{\bar{\nu}} \leqslant(b-a) /[4(1+\delta)], \forall n \in \mathbb{N}$.

(c) For every $n \geqslant n_{1}$, there exists $\Sigma_{n}^{H}$, a subset of $T_{n}^{H}$, having measure $(1+\delta) \varepsilon_{n}^{\bar{v}}$. Define the absolutely continuous functions $t_{n}(s)=a+\int_{a}^{s} t_{n}^{\prime}(\tau) \mathrm{d} \tau$ by setting

$$
t_{n}^{\prime}(s)= \begin{cases}1+\left[\frac{\left|x_{n}^{\prime}(s)\right|}{\bar{v}}-1\right], & s \in S_{n}^{\bar{v}}, \\ 1-\frac{1}{1+\delta}, & s \in \Sigma_{n}^{H}, \\ 1, & \text { otherwise }\end{cases}
$$

each $t_{n}$ is an invertible map from $[a, b]$ onto itself.

(d) From the definition of $t_{n}^{\prime}$ we have that

$$
\begin{aligned}
\int_{a}^{b} L & \left(x_{n}(s), \frac{x_{n}^{\prime}(s)}{t_{n}^{\prime}(s)}\right) t_{n}^{\prime}(s) \mathrm{d} s-\int_{a}^{b} L\left(x_{n}(s), x_{n}^{\prime}(s)\right) \mathrm{d} s \\
= & \int_{S_{n}^{\bar{v}}} L\left(x_{n}(s), \bar{v} \frac{x_{n}^{\prime}(s)}{\left|x_{n}^{\prime}(s)\right|}\right) \frac{\left|x_{n}^{\prime}(s)\right|}{\bar{v}} \mathrm{~d} s-\int_{S_{n}^{\bar{v}}} L\left(x_{n}(s), x_{n}^{\prime}(s)\right) \mathrm{d} s \\
& +\int_{\Sigma_{n}^{H}} L\left(x_{n}(s),(1+\delta) x_{n}^{\prime}(s)\right) \frac{1}{1+\delta} \mathrm{d} s-\int_{\Sigma_{n}^{H}} L\left(x_{n}(s), x_{n}^{\prime}(s)\right) \mathrm{d} s
\end{aligned}
$$

We wish to estimate the above integrals. Since $\Sigma_{n}^{H} \subset T_{n}^{H}$, we easily obtain

$$
\int_{\Sigma_{n}^{H}}\left[L\left(x_{n}(s),(1+\delta) x_{n}^{\prime}(s)\right) \frac{1}{1+\delta}-L\left(x_{n}(s), x_{n}^{\prime}(s)\right)\right] \mathrm{d} s \leqslant(1+\delta) \varepsilon_{n}^{\bar{v}} \mu .
$$

To conclude the estimate we have to consider separately the convex and the differentiable case.

(e) (Convex case) The choice of $p$ implies that

$$
\begin{aligned}
& L\left(x_{n}(s), \bar{v} \frac{x_{n}^{\prime}(s)}{\left|x_{n}^{\prime}(s)\right|}\right) \frac{\left|x_{n}^{\prime}(s)\right|}{\bar{v}}-L\left(x_{n}(s), x_{n}^{\prime}(s)\right) \\
& \quad \leqslant-\left[\frac{\left|x_{n}^{\prime}(s)\right|}{\bar{v}}-1\right] L^{*}\left(x_{n}(s), p\left(x_{n}(s), \bar{v} \frac{x_{n}^{\prime}(s)}{\left|x_{n}^{\prime}(s)\right|}\right)\right)
\end{aligned}
$$

for every $s \in S_{n}^{\bar{\nu}}$.

(e) (Differentiable case) The Mean Value Theorem implies that there exists $\alpha_{n}(s) \in$ $\left[0,\left|x_{n}^{\prime}(s)\right| / \bar{v}-1\right]$ such that 


$$
\begin{aligned}
L( & \left.x_{n}(s), \bar{v} \frac{x_{n}^{\prime}(s)}{\left|x_{n}^{\prime}(s)\right|}\right) \frac{\left|x_{n}^{\prime}(s)\right|}{\bar{v}}-L\left(x_{n}(s), x_{n}^{\prime}(s)\right) \\
= & -\left[\frac{\left|x_{n}^{\prime}(s)\right|}{\bar{v}}-1\right]\left[\left\langle\nabla_{\xi} L\left(x_{n}(s), \frac{x_{n}^{\prime}(s)}{1+\alpha_{n}(s)}\right), \frac{x_{n}^{\prime}(s)}{1+\alpha_{n}(s)}\right\rangle\right. \\
& \left.-L\left(x_{n}(s), \frac{x_{n}^{\prime}(s)}{1+\alpha_{n}(s)}\right)\right] \\
= & -\left[\frac{\left|x_{n}^{\prime}(s)\right|}{\bar{v}}-1\right] L^{*}\left(x_{n}(s), \nabla_{\xi} L\left(x_{n}(s), \frac{x_{n}^{\prime}(s)}{1+\alpha_{n}(s)}\right)\right)
\end{aligned}
$$

for every $s \in S_{n}^{\bar{\nu}}$.

(f) Since both $\left|\bar{v} x_{n}^{\prime}(s) /\right| x_{n}^{\prime}(s)|| \geqslant \bar{v}$ and $\left|x_{n}^{\prime}(s)\right| /\left(1+\alpha_{n}(s)\right) \geqslant \bar{v}$, by the definition of $M(\bar{v})$ we obtain

$$
\int_{S_{n}^{\bar{v}}} L\left(x_{n}(s), \bar{v} \frac{x_{n}^{\prime}(s)}{\left|x_{n}^{\prime}(s)\right|}\right) \frac{\left|x_{n}^{\prime}(s)\right|}{\bar{v}} \mathrm{~d} s-\int_{S_{n}^{\bar{v}}} L\left(x_{n}(s), x_{n}^{\prime}(s)\right) \mathrm{d} s \leqslant-\varepsilon_{n}^{\bar{v}} M(\bar{v}),
$$

hence our estimate becomes: $n \geqslant n_{1}$ implies that

$$
\int_{a}^{b} L\left(x_{n}(s), \frac{x_{n}^{\prime}(s)}{t_{n}^{\prime}(s)}\right) t_{n}^{\prime}(s) \mathrm{d} s-\int_{a}^{b} L\left(x_{n}(s), x_{n}^{\prime}(s)\right) \mathrm{d} s \leqslant \varepsilon_{n}^{\bar{v}}[-M(\bar{v})+(1+\delta) \mu] \leqslant 0 .
$$

(g) The conclusion of (f) proves the theorem; in fact, defining $\bar{x}_{n}=x_{n} \circ s_{n}$, where $s_{n}$ is the inverse of the function $t_{n}$, we obtain, by the change of variable formula [7], that $\left\{\bar{x}_{n}\right\}_{n \geqslant n_{1}}=\left\{x_{n} \circ s_{n}\right\}_{n \geqslant n_{1}}$ is a minimizing sequence, since

$$
\begin{aligned}
\int_{a}^{b} L\left(\bar{x}_{n}(t), \bar{x}_{n}^{\prime}(t)\right) \mathrm{d} t & =\int_{a}^{b} L\left(\bar{x}_{n}\left(t_{n}(s)\right), \frac{\mathrm{d} \bar{x}_{n}}{\mathrm{~d} t}\left(t_{n}(s)\right)\right) t_{n}^{\prime}(s) \mathrm{d} s \\
& =\int_{a}^{b} L\left(x_{n}(s), \frac{x_{n}^{\prime}(s)}{t_{n}^{\prime}(s)}\right) t_{n}^{\prime}(s) \mathrm{d} s \leqslant \int_{a}^{b} L\left(x_{n}(s), x_{n}^{\prime}(s)\right) \mathrm{d} s .
\end{aligned}
$$

Moreover, we claim that $\bar{x}_{n}$ are Lipschitzian functions, with the same Lipschitz constant $\Lambda=(1+1 / \delta) \bar{v}$. In fact, consider the equality $\bar{x}_{n}^{\prime}\left(t_{n}(s)\right)=x_{n}^{\prime}(s) / t_{n}^{\prime}(s)$ and fix $s$ where $t_{n}^{\prime}(s)$ exists; we obtain

$$
\left|\frac{\mathrm{d} \bar{x}_{n}}{\mathrm{~d} t}\left(t_{n}(s)\right)\right| \begin{cases}=\bar{v}, & s \in S_{n}^{\bar{\nu}} \\ \leqslant(1+1 / \delta) \bar{v}, & s \in \Sigma_{n}^{H} \\ \leqslant \bar{v}, & \text { otherwise }\end{cases}
$$

hence, at almost every point $t_{n}(s)$, the norm of the derivative of $\bar{x}_{n}$ is bounded by $\Lambda$. This completes the proof. 


\section{REFERENCES}

[1] A. Cellina, Reparametrizations and the non-occurrence of the Lavrentiev phenomenon in the autonomous case of the calculus of variations, Preprint, 2001.

[2] A. Cellina, The classical problem of the calculus of variations in the autonomous case: Relaxation and Lipschitzianity of solutions, Trans. Amer. Math. Soc., submitted for publication.

[3] A. Cellina, G. Treu, S. Zagatti, On the minimum problem for a class of non-coercive functionals, J. Differential Equations 127 (1996) 225-262.

[4] L. Cesari, Optimization, Theory and Applications, Springer-Verlag, New York, 1983.

[5] F.H. Clarke, R.B. Vinter, Regularity properties of solutions to the basic problem in the calculus of variations, Trans. Amer. Math. Soc. 289 (1985) 73-98.

[6] I. Ekeland, R. Temam, Convex Analysis and Variational Problems, North-Holland, Amsterdam, 1976.

[7] J. Serrin, D.E. Varberg, A general chain rule for derivatives and the change of variable formula for the Lebesgue integral, Amer. Math. Monthly 76 (1969) 514-520. 